\title{
Moneyball in Ophthalmology: Historic Failures in 0phthalmology Innovations and How to Overcome them
}

\author{
Kenneth Lu*, MD \\ Doheny Eye Institute, USA \\ Submission: March 25, 2019; Published: April 01, 2019 \\ *Corresponding author: Kenneth Lu, Doheny Eye Institute/ UCLA, 622 W Duarte Rd, Suite 101, Arcadia, CA 91007, USA
}

Keywords: Accommodative IOL; Innovations; R\&D; Moneyball; Helmholtz theory; Sabermetrics

\begin{abstract}
Opinion
Don't get me wrong. Ophthalmology is one of the most successfully innovative fields out there. But the road to success has also been littered with some spectacular failures, for instance, Synchrony IOL, Sunrise Laser Thermal Keratoplasty (LTK), Conductive Keratoplasty (CK), and arguably femtosecond cataract surgery. Collectively, these have cost the industry billions of dollars to purchase but have not reaped positive returns. To understand these failures, one must understand the innovations pathway, which most often starts with grassroots efforts that must overcome a lack of resources in order to build value and process so eventually can be sold to one of the industry giants.
\end{abstract}

The problem is that these industry giants are not in on the ground floor. They are not familiar enough with the ins and outs of the tech development history. They are presented with packaged up new techs where potential is maximized but problems and risks are minimized. It's the same reason we often pick bad residents. The interview process is woefully inadequate in selecting hard working, ethical, efficient residents, because it is not set up to evaluate for those qualities.

A perfect example of this is the accommodative IOL market. We all know the accepted mechanism of accommodation by Helmholtz's Theory [1]. It says that accommodation occurs when the ciliary muscle contracts, causing circumferential constriction, which relaxes the zonules, thereby allowing the natural crystalline lens to assume it's more rounded resting shape. Conversely, during dis-accommodation, the ciliary muscles relax, causing circumferential expansion, which tightens the zonules and subsequently flattens the lens. The driving force is the natural elasticity of the lens. The zonules simply connect the lens to the ciliary body. There is no such thing as ciliary push, i.e. when ciliary muscle contracts, it does not push on the lens. It's not even in contact with the lens. Yet, there are dozens of companies developing accommodative IOLs based on the concept of ciliary push. One even slipped through the FDA process. To date, there is scant (to put it kindly) evidence that "accommodative" IOL actually moves inside the eye.

When the industry giants look at these IOLs, they focus on the clinical data, which may represent pseudo-accommodation, and forget the fundamental design flaw that defies accepted theory of the mechanism. What is needed, is for these corporations to set accommodative IOL as a goal, a holy grail, and start their own development programs from scratch. They can source lots of sensible and logical ideas and patents for cheap, or come up with their own designs, and devote adequate resources to develop these into workable, commercialize products. At the pre-prototype stage, patents can be licensed for hundreds of thousands to single-digit millions. Even if they buy 100 of these ideas, it would still be much cheaper than the $\$ 400$ million spent on Synchrony [2]. The rest could be spent on development, and they'd still be ahead. And because a true accommodative IOL market is in the billions, even if only 1 out of 100 idea makes it, they would all still be smart investments.

This brings me to the title, Moneyball in Ophthalmology. Moneyball [3] was a 2003 book (made into a movie with Brad Pitt) about the Oakland A's unconventional (at the time; now all teams use sabermetrics) method of choosing players, relying on player statistics rather than their raw abilities. They were able to go far into the playoffs with much less money than marquee teams ( $\$ 44$ million as compared to NY Yankees' $\$ 125$ million in 2002). They were in essence smarter and more efficient with their money. The same system of buying undervalued players based on sound statistics could apply to ophthalmology, where industry giants invests much earlier in the innovative process at much lower costs, 
spread the investments around to reduce risk instead of having all their eggs in one basket. This allows them to maintain control, quality, and budget.

By getting earlier, several things happen. Funding risk for the development team is removed, which eliminates much now unnecessary fund-raising efforts. Conflict of interest is removed, as no salesmanship is needed anymore. Industry can now truthfully and objectively examine their prospects. Efficiency is established. Continuity is ensured. Budget is better controlled. When this true $R \& D$ department is set up, there can be also lots of centralized savings, as much cross-over in product development can utilize the same resources within the company.

This in fact, is what the general public believes is happening in large corporation R\&D departments, but over the years, these have gone by the wayside. It's time to bring back a true R\&D culture in ophthalmic corporations. Diligence and accountability are needed to ensure efficiency and avoid wastage. The math however points to this being a viable method of industry tech development. It is frankly more fun too, to be involved from the get-go. Who knows, maybe one day a movie with Ryan Gosling directly by Ridley Scot will be made about how a true accommodative IOL was started by a simple yet revolutionary idea of Moneyball in Ophthalmology.

\section{Conflict of Interest}

The author holds a patent on an accommodative IOL design.

\section{References}

1. https://en.wikipedia.org/wiki/Accommodation_(eye)

2. https://www.biospace.com/article/releases/abbott-laboratoriescompletes-visiogen-inc-acquisition-/

3. https://en.wikipedia.org/wiki/Moneyball 